\title{
Strongly Directed Electron Emission in Non-Sequential Double Ionization of Ne by Intense Laser Pulses
}

\author{
R. Moshammer ${ }^{1 *}$, J. Ullrich $^{1}$, B. Feuerstein ${ }^{1}$, D. Fischer ${ }^{1}$, A. Dorn ${ }^{1}$, \\ C.D. Schröter ${ }^{1}$, J.R Crespo Lopez-Urrutia ${ }^{1}$, C. Höhr ${ }^{1}$ \\ ${ }^{1}$ Max-Planck-Institut für Kernphysik, Saupfercheckweg 1, D-67119 \\ Heidelberg, Germany
}

\begin{abstract}
H. Rottke ${ }^{2}$, C. Trump ${ }^{2}$, M. Wittmann ${ }^{2}$, G. Korn ${ }^{2}$, K. Hoffmann ${ }^{2}$, W. Sandner ${ }^{2}$ ${ }^{2}$ Max-Born-Institut, Max-Born-Str. 2a, D-12489 Berlin, Germany *e-mail: Moshammer@mpi-hd.mpg-de
\end{abstract}

\begin{abstract}
Double ionization of $\mathrm{Ne}$ by $25 \mathrm{fs}, 1.0 \mathrm{PW} / \mathrm{cm}^{2}$ laser pulses has been explored in a kinematically complete experiment using a "Reaction Microscope". Electrons are found to be emitted into a narrow cone along the laser polarization $(\varepsilon)$, much more confined than for single ionization, with a broad maximum in their energy distribution along $\varepsilon$. Correlated momentum spectra show both electrons being ejected into the same hemisphere, in sharp contradiction to predictions based on field-free (e,2e) recollision dynamics, but, in overall agreement with recent semi-classical calculations for He.
\end{abstract}

PACS numbers: $32.80 . \mathrm{Rm}, 32.80 . \mathrm{Fb}, 32.90 .+\mathrm{a}, 42.50 . \mathrm{Hz}$ 
Double ionization of atoms is one of the most fundamental processes when intense laser fields interact with matter, and, at the same time, one of the most challenging problems in atomic physics, theoretically as well as experimentally. Whereas single ionization is well understood in the framework of the "single active electron model" (SAE), the measured yields of double ionization exceed those theoretically expected assuming a sequence of individual and independent single ionization events by many orders of magnitudes at an intermediate intensity regime. Undoubtedly, this "nonsequential" (NS) contribution to double ionization is a consequence of the electron-electron interaction, the underlying mechanism, however, remained unclear over many years (for reviews see $[1,2]$ ).

Only recently, a new generation of differential measurements [3,4] along with various quantum [5-10] as well as classical calculations [11,12] provided convincing evidence that the so-called "rescattering" or recollision scenario one of the three NS double ionization pathways (beyond collective tunneling, and shake-off) which have been proposed in the past - is the dominating mechanism. Here, one electron is ionized via tunneling, accelerated in the oscillating laser field and, under certain conditions, driven back to its parent ion knocking out another electron. It was demonstrated, that the kinematics of this dynamical process is the only one that leads to recoil-ion momentum distributions with a "double-hump" structure as observed in the experiments [13]. Even before, results of experiments on harmonic generation [1] and, in particular, the disappearance of NS-ionization when circularly light is used [14] were strongly in favor of the rescattering model. Thus, it is generally accepted presently that rescattering is the dominant NS double ionization 
mechanism in an intermediate intensity regime. On the other side, however, the underlying dynamics during recollision, i.e. the electron impact ionization (e,2e) dynamics in the presence of the laser field, its intensity or pulse-time dependence is far from being understood.

Consequently, a series of recent experiments have addressed this question on different levels of sophistication. Ratios of double to single ionization and electron energy distributions for double ionization (partly integrated over emission angles for low electron energies) have been reported [15-18] along with experiments, where one momentum component of one emerging electron was measured in coincidence with the full momentum vector of the recoiling target ion providing information about the correlated momenta of both emitted electrons along the laser polarization direction $[19,20]$. Here, for NS double ionization of Ar by 0.38 and $0.2 \mathrm{PW} / \mathrm{cm}^{2}, 780 \mathrm{~nm}$ pulses at a pulse length of 220 and $25 \mathrm{fs}$, respectively, detailed information on the momentum sharing between both ejected electrons was obtained, which sensitively depends on the recollision dynamics. In both experiments, the two electrons were found to be predominantly emitted into the same hemisphere in agreement with the kinematical constraints set by the classical rescattering model. In addition, however, a considerable fraction was observed to be emitted back-to-back, which has been interpreted as being due to excitation during rescattering [20] with subsequent and independent field (tunnel) ionization of the second electron. Measuring in addition the transverse momentum of one of the electrons, the recollision dynamics was explored in even more detail. Setting severe constraints on the transverse motion of the electrons, signatures of the 
final-state repulsion between the two emitted electrons along the polarization direction were observed [21,22].

For lighter target atoms, where excitation can be expected to be less important and, thus, the recollision dynamics should be less obscured by the target electronic structure, no experimental data on correlated electron emission for strong-field double ionization have been reported up to now because of enormous experimental complications. Here, the comparison with theory, completely neglecting any influence of the atomic structure, should become much more reliable. Quantum mechanical S-Matrix calculations [8] indeed predict the correlated electron momentum distributions to depend sensitively on the details of the recollision process: Different approximations used to account for the electron-electron correlation in double ionization of $\mathrm{Ne}$ led to completely different two-electron momentum patterns. If a simplified contact potential is applied to describe the interaction between the colliding electrons, they both appear with similar momenta along the polarization direction in the final state. In contrast, if a more realistic Coulomb form factor is used a quite asymmetric electron momentum sharing is predicted. Applying a three-dimensional semi-classical model Fu et al. [12] have obtained a quite symmetric momentum sharing for $1 \mathrm{PW} / \mathrm{cm}^{2}$ pulses interacting with $\mathrm{He}$ atoms with both electrons having most likely similar or even equal momenta along the polarization direction. Fully quantummechanical theoretical treatments solving the three-dimensional Schrödinger equation [9] are only feasible at higher photon frequencies with present computer capabilities. Hence, the correlated two-electron dynamics in the 
presence of the laser field occurring during rescattering essentially remained in the dark.

In this Letter, highly differential information on strong-field $\left(1 \mathrm{PW} / \mathrm{cm}^{2}\right)$ non-sequential double ionization of $\mathrm{Ne}$ is reported, obtained in a kinematically complete experiment with a reaction microscope. Energy- and angle-resolved electron spectra, covering the major part of the final-state momentum space, are presented in addition to two-electron momentum distributions along the polarization direction $(\varepsilon)$. Electrons are found to be strongly directed along $\varepsilon$ with similar momenta, in severe contradiction to field-free (e,2e) recollision kinematics [8] but in qualitative agreement with classical predictions [12]. Back-to-back emission of both electrons is extremely unlikely for $\mathrm{Ne}$ in sharp contrast to the Ar results.

The experiment was performed at the Max-Born Institute in Berlin using a Kerr-lens mode locked Ti:Sapphire laser at $800 \mathrm{~nm}$ wavelength with a repetition rate of $1 \mathrm{kHz}$. By focussing the $25 \mathrm{fs}$ (FWHM) light pulses to a spot of $10 \mu \mathrm{m}$ (FWHM) diameter into an ultrahigh vacuum chamber $\left(2 \cdot 10^{-11}\right.$ torr $)$ pulse peak intensities of $1.0 \mathrm{PW} / \mathrm{cm}^{2}$ were reached. At its focus the laser beam was crossed by a well collimated low-density $\left(10^{8}\right.$ atoms $\left./ \mathrm{cm}^{3}\right)$ supersonic $\mathrm{Ne}$ jet resulting in an interaction volume of $50 \mu \mathrm{m}$ length and $10 \mu \mathrm{m}$ diameter. Using a reaction-microscope, which has been described in detail in [2,20,22], the ionic charge as well as the coincident momentum vectors of both, ions and electrons, were recorded with a momentum resolution of $\Delta \mathrm{p} \approx 0.1$ a.u.. Intensity fluctuations were monitored during the experiment and kept below $5 \%$. The stability of all experimentally relevant parameters was maintained throughout the beam-time of several days. 
In Fig.1 the longitudinal momentum (i.e. the component along the laser polarization axis) of one electron is plotted versus that of the other electron without any restriction on their transverse momenta. A strongly correlated electron motion is observed in a way that both electrons are preferentially emitted into the same hemisphere with very similar momenta. Different from all previous results for Ar targets [19-22], back-to-back-emission is essentially absent, i.e. only few events are observed where both electrons escape into opposite directions, confirming the expectation that recollisional excitation and subsequent field ionization is less important for lighter targets. Moreover, one might now inspect whether the measured distribution is compatible with the recollision kinematics (see also [20]). For a certain tunneling phase of the first electron $\omega_{0}$, recollision happens at a well defined recollision energy $E^{\text {recoll }}$ and phase $\omega t_{1}$. The latter results in well defined and

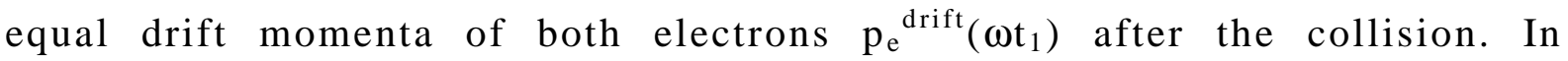
addition, they can share the excess energy $E^{e x}=E^{\text {recoll }}-I_{P}\left(I_{P}\right.$ : ionization potential). Neglecting the transverse energies of the electrons, which will be shown to be small, energy conservation $\mathrm{p}_{\mathrm{e} 1 \|^{2}}+\mathrm{p}_{\mathrm{e} 2 \|^{2}}=2 \mathrm{E}^{\mathrm{ex}}$ during rescattering yields a circle with radius $\sqrt{2 E^{e x}}$ around the points $\left( \pm \mathrm{p}_{\mathrm{e} 1}{ }^{\mathrm{drift}}\left(\omega \mathrm{t}_{1}\right), \pm \mathrm{p}_{\mathrm{e} 2}{ }^{\mathrm{drift}}\left(\omega \mathrm{t}_{1}\right)\right)$ as maximum drift momentum combinations. Considering all phases, the full lines in Fig.1 are obtained indicating the classical boundary for rescattering. The comparison with the experimental data impressively shows that nearly all events are within these boundaries.

While practically all events are compatible with the kinematical boundaries for recollision the correlation pattern itself, however, with many 
electrons of quite similar longitudinal momenta is in contradiction with the predictions based on field-free electron-impact (e,2e) ionization dynamics, which becomes actually more obvious at higher intensities [8]. In accordance with the fact that unequal energy sharing between projectile and ionized electron is by far the most likely configuration in field-free $(\mathrm{e}, 2 \mathrm{e})$ at not too low impact energy, unequal final momenta are predicted as well for NS double ionization in laser fields in striking contrast to the experimental results. Several other predictions, using a hard core form factor for the electron collision [8] or solving the one-dimensional time-dependent Schrödinger equation with correlated electrons are in poor agreement with the experiment. Only recent semi-classical calculations [12] were able to predict a similar pattern as observed. There, the first electron is set free with a velocity distribution following from tunneling theory and propagated classically in the combined fields of the laser and the ionic core. The remaining $\mathrm{He}^{+}$ion is also modeled classically, with the electron in a micro-canonical distribution in the intermediate state. Thus, within the classical approximation, all interactions are correctly taken into account in all three dimensions.

To further elucidate electron emission in NS double ionization of $\mathrm{Ne}$, electron energy distributions are shown in Fig.2 for single as well as double ionization integrated over all emission angles. At the present laser intensity of 1.0 $\mathrm{PW} / \mathrm{cm}^{2}$ the Keldysh parameter [23] is $\gamma=0.30$, indicating that single ionization mainly proceeds via tunneling [24]. Since tunneling ionization is most likely close to the maximum of the electric field $\vec{F}(t)=\vec{F}_{o}(t) \cdot \sin (\omega t)$ the final drift momentum $p^{\text {drift }}\left(t_{0}\right)=1 / \omega \cdot F_{0}\left(t_{0}\right) \cos \left(\omega t_{0}\right)$ is typically small. Thus, 
structure less electron spectra are expected, peaked at low kinetic energies in agreement with experiment.

In comparison, the electron energy distribution in coincidence with double ionization is significantly less steep, extending to much higher energies. Similar observations have been reported recently for emission along the polarization axis for $\mathrm{Xe}$, Ar, and He targets [15-17]. Larger electron energies are in qualitative agreement with the rescattering scenario, since recollision most likely happens at a phase close to a zero crossing of the oscillating electric field. Hence, both electrons accumulate large drift momenta or, equivalently, large final kinetic energies of up to $E_{\max }=F_{0}^{2} /\left(2 \omega^{2}\right)=2 U_{P}=120 \mathrm{eV}$ for the present laser intensity. Obviously, inspecting doubly differential energy spectra for different emission angles $\vartheta$ with respect to the polarization in Fig.3, this value sets an upper limit for the electron energies for emission along $\varepsilon$. Here the differences in the spectra between single and double ionization are even more significant: Whereas the distribution strongly peaks at small energies for $\mathrm{Ne}^{1+}$ production, a broad maximum around $30 \mathrm{eV}$ is observed for double ionization. Such a behavior has not been observed in the previous measurements [15-17].

In contrast to the strongly different energy distributions for single and double ionization the electron angular distributions integrated over all electron energies are very similar as illustrated in Fig.4. In both cases, as might have been expected from the drift motion, electrons are preferentially emitted along the polarization axis. Most surprisingly however, the angular distribution is significantly narrower for double ionization, intuitively 
contradicting the recollision scenario: During the $(\mathrm{e}, 2 \mathrm{e})$-like rescattering event the returning projectile electron as well as the ionized electron can gain considerable momenta in the transverse direction of up to $p_{\perp}^{\max }<\sqrt{2 \cdot\left(3.17 U_{P}-I_{p}\right)}$ which, for the present laser intensity, can be as large as the maximum drift momentum of $2 \cdot \sqrt{U_{P}}$. Hence, a substantial broadening of the angular emission would be expected and even electron emission angles of up to $\vartheta=45^{\circ}$ might occur.

Staying within the recollision scenario, the sharply peaked angular distribution for electron emission in double ionization necessarily leads to the conclusion that large transverse electron momenta are unlikely to appear. Obviously, the dominant contribution to NS double ionization of $\mathrm{Ne}$ comes from nonviolent, soft (e,2e) collisions with small energy and momentum transfers between the colliding partners, where the external field substantially assists to overcome the binding energy of the second electron, i.e. at a most likely recollision phase where the field is not zero. Then, the ponderomotive force, acting along the polarization axis, dictates the emission characteristics of electrons being "just" promoted into the continuum during recollision, resulting in a strongly directed, jet-like electron ejection with very similar longitudinal momenta, significantly below the maximum drift momentum. Such an interpretation is supported by recent measurements at laser intensities below the classical threshold for (e,2e) during recollision, where the doublehump structure for Ne is still observed [25].

In summary, the first kinematically complete experimental data on NS double ionization in intense laser pulses have been presented. Strongly 
directed and correlated jet-like electron emission was observed. Even though the experimental data are in accordance with the kinematics of the rescattering mechanism, the attempt to interpret the data based on the well known dynamics of field-free electron impact ionization fails. On the contrary, one is led to the conclusion, that the laser field is crucially required to obtain double ionization: At a phase were the field is not zero low-energy electrons are created during recollision whose kinematics is then dictated by the external field.

The experiments were supported by the Deutsche Forschungsgemeinschaft (DFG, RO 1929) and the Leibniz-Program of DFG. Support from GSI is gratefully acknowledged. We are grateful to $\mathrm{R}$. Dörner and W. Becker for numerous discussions. 


\section{Figure Captions}

Fig.1: Correlated two-electron momentum distributions along the laser polarization axis for electrons ejected in double ionization of $\mathrm{Ne}$ by $25 \mathrm{fs}$, linearly polarized, $800 \mathrm{~nm}$ laser pulses at a peak intensity of $1.0 \mathrm{PW} / \mathrm{cm}^{2}$. The data are integrated over the momentum components perpendicular to the polarization. The solid curve denotes the upper limit of correlated electron momenta compatible with the classical rescattering model. The $\mathrm{z}$-axis is on a logarithmic scale with box sizes corresponding to the respective intensity.

Fig.2: Electron energy distributions integrated over all emission angles for single and double ionization of $\mathrm{Ne}$ for the same laser parameters as in Fig.1. In the case of double ionization the distribution of one (typical) of the two electrons is shown. The $\mathrm{Ne}^{2+}$-counts have to be divided by a factor of 10 to recover the experimental double to single ionization ratio.

Fig.3: Electron energy distributions for fixed emission angles $\vartheta$ (integrated over $\Delta \vartheta= \pm 5^{\circ}$ ) with respect to the polarization axis for double ionization of Ne. The distribution of one (typical) of the two electrons is shown.

Fig.4: Angular distributions of electrons ejected in single and double ionization of $\mathrm{Ne}$. The number of registered electrons per solid angle $\sin \vartheta \mathrm{d} \vartheta$ is plotted versus $\vartheta$. Intensities are the same as in Fig. 1. 


\section{References}

[1] M. Protopapas, C.H. Keitel and P.L. Knight, Rep. Prog. Phys. 60, 389 (1997)

[2] R. Dörner et al., Adv. In At., Mol, and Opt. Physics 48 (2002), in print

[3] Th. Weber et al., Phys. Rev. Lett. 84, 443 (2000)

[4] R. Moshammer et al., Phys. Rev. Lett. 84, 447 (2000)

[5] A. Becker and F.H.M. Faisal, Phys. Rev. Lett. 84, 3546 (2000)

[6] M. Lein, E.K.U. Gross, and V. Engel, Phys. Rev. Lett. 85, 4707 (2000)

[7] S.L. Haan et al., Optics Express 7, 29 (2000)

[8] S.P. Goreslavskii, S.V. Popruzhenko, R. Kopold, and W. Becker, Phys. Rev. A 64, 053402 (2001)

[9] J. S. Parker, et al., J. Phys. B 33, L691 (2001); 34, L69 (2001)

[10] R. Kopold et al., Phys. Rev. Lett. 85, 3781 (2000)

[11] K. Sacha and B. Eckhard, Phys. Rev. A 63, 043414 (2001)

[12] L.B. Fu, J. Liu, and S.G. Chen, Phys. Rev. A 65, 021406 (2002)

[13] B. Feuerstein, R. Moshammer, and J. Ullrich, J. Phys. B 33, L823 (2001)

[14] D.N. Fittinghoff et al., Phys. Rev. A 49, 2174 (1994)

[15] B. Witzel, N.A. Papadogiannis, and D. Charalambidis, Phys. Rev. Lett. 85, $2268(2000)$

[16] E.R. Peterson and P.H. Bucksbaum, Phys. Rev. A 64, 053405 (2001)

[17] R. Lafon et al., Phys. Rev. Lett. 86, 2762 (2001)

[18] V.R. Bhardwaj et al., Phys. Rev. Lett. 86, 3522 (2001)

[19] Th. Weber et al., Nature. 404, 608 (2000)

[20] B. Feuerstein et al., Phys. Rev. Lett. 87, 043003-1 (2001) 
[21] M. Weckenbrock et al., J. Phys. B 34, L449 (2001)

[22] R. Moshammer et al., Phys. Rev. A 65, 035401 (2002)

[23] L.V. Keldysh, Zh. Eksp. Teor. Fiz. 47, 1945 (1964)

[24] N.B. Delone, and V.P. Krainov, Physics - Uspekhi 41 (5) 469 (1998)

[25] E. Eremina et al., Phys. Rev. Lett. (2002), submitted 


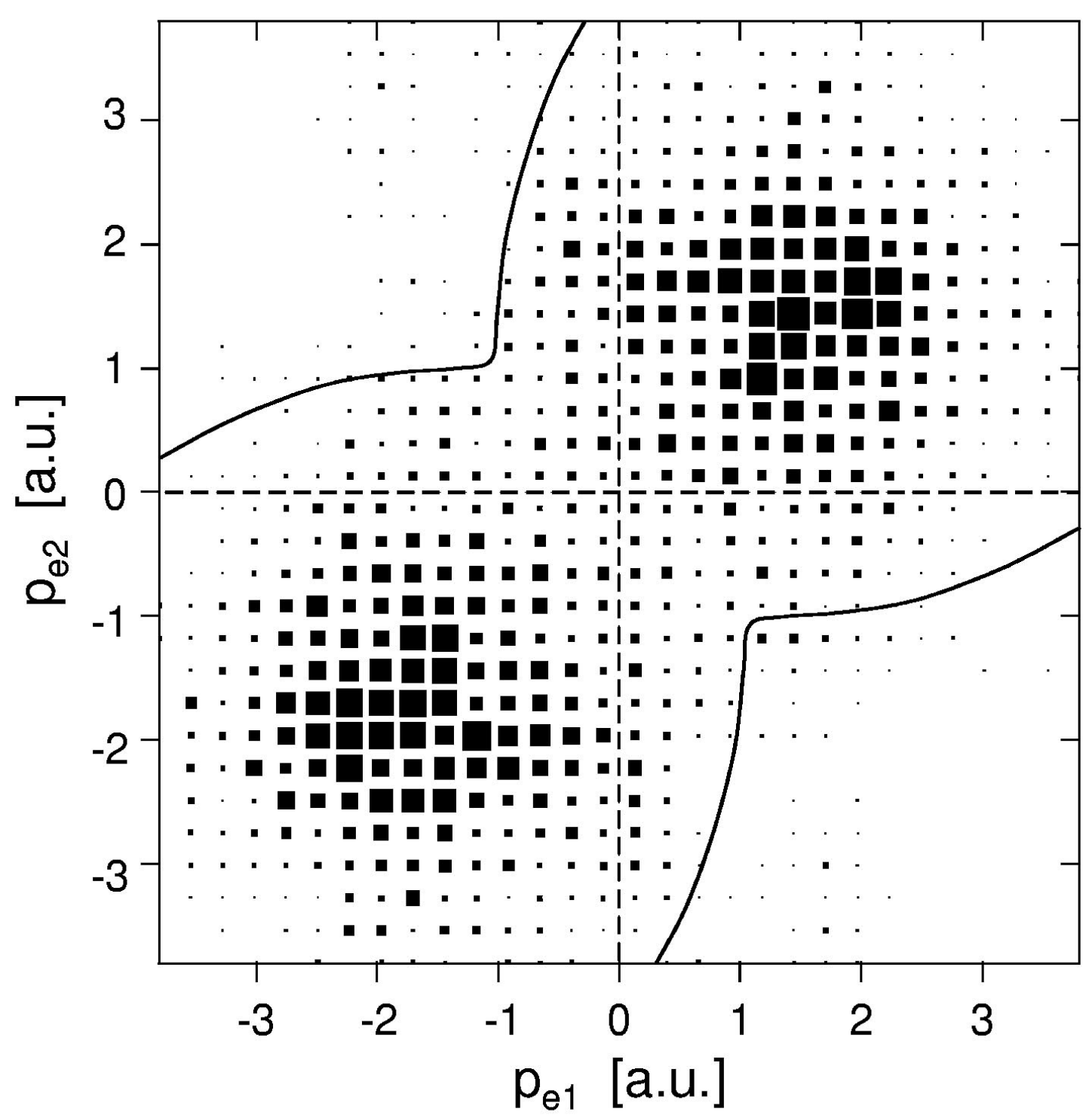

Figure 1 


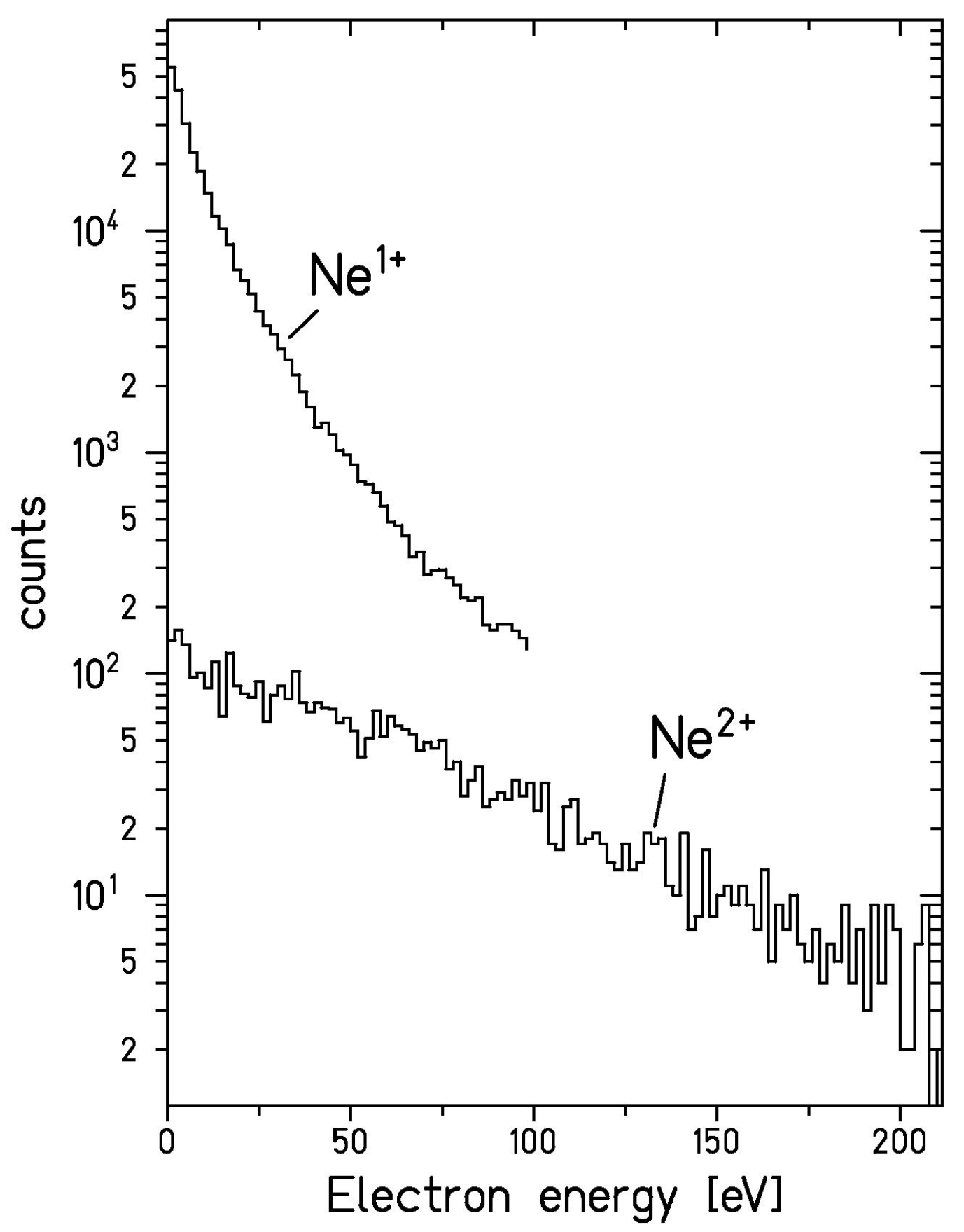

Figure 2 


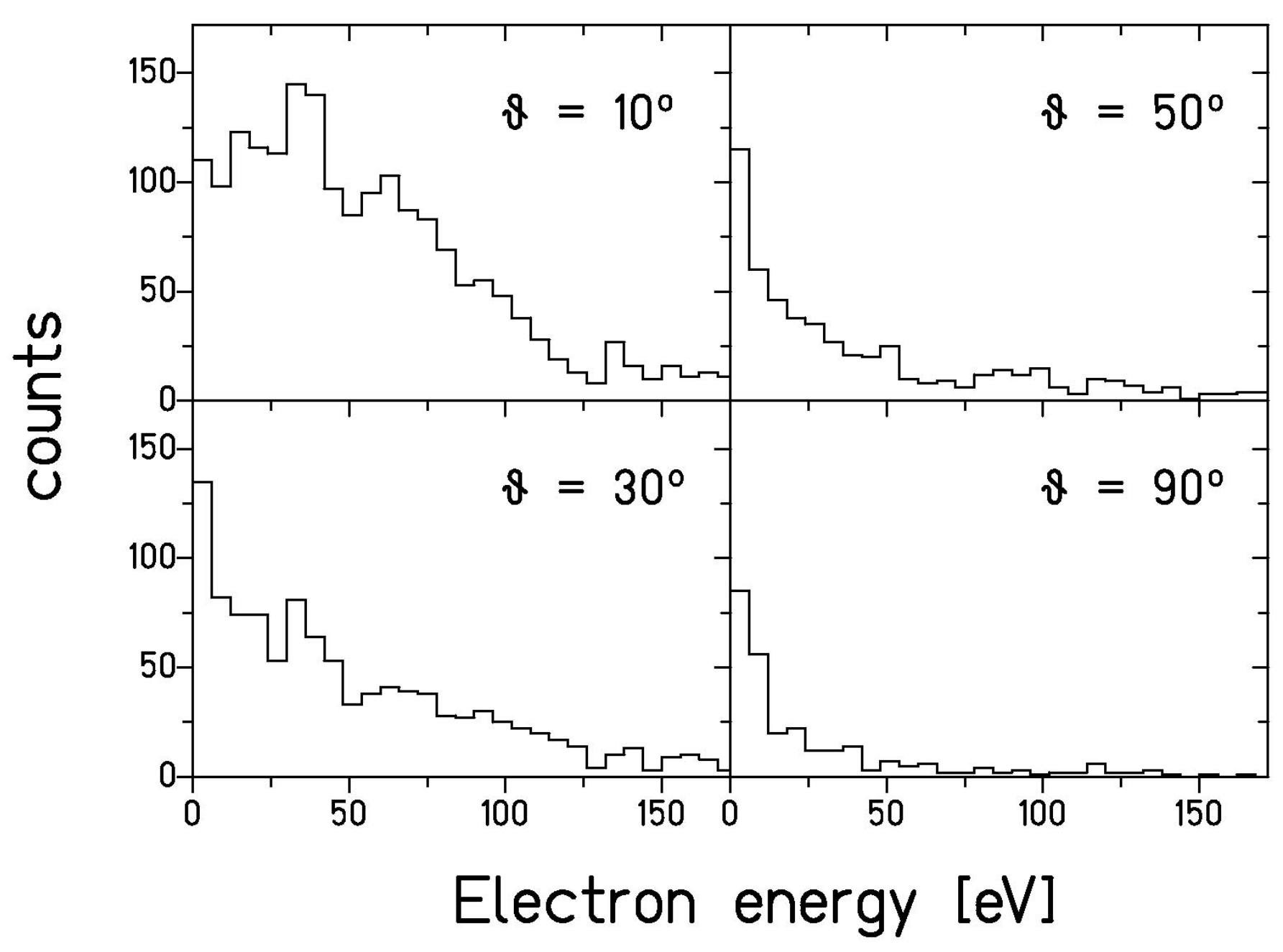

Figure 3 


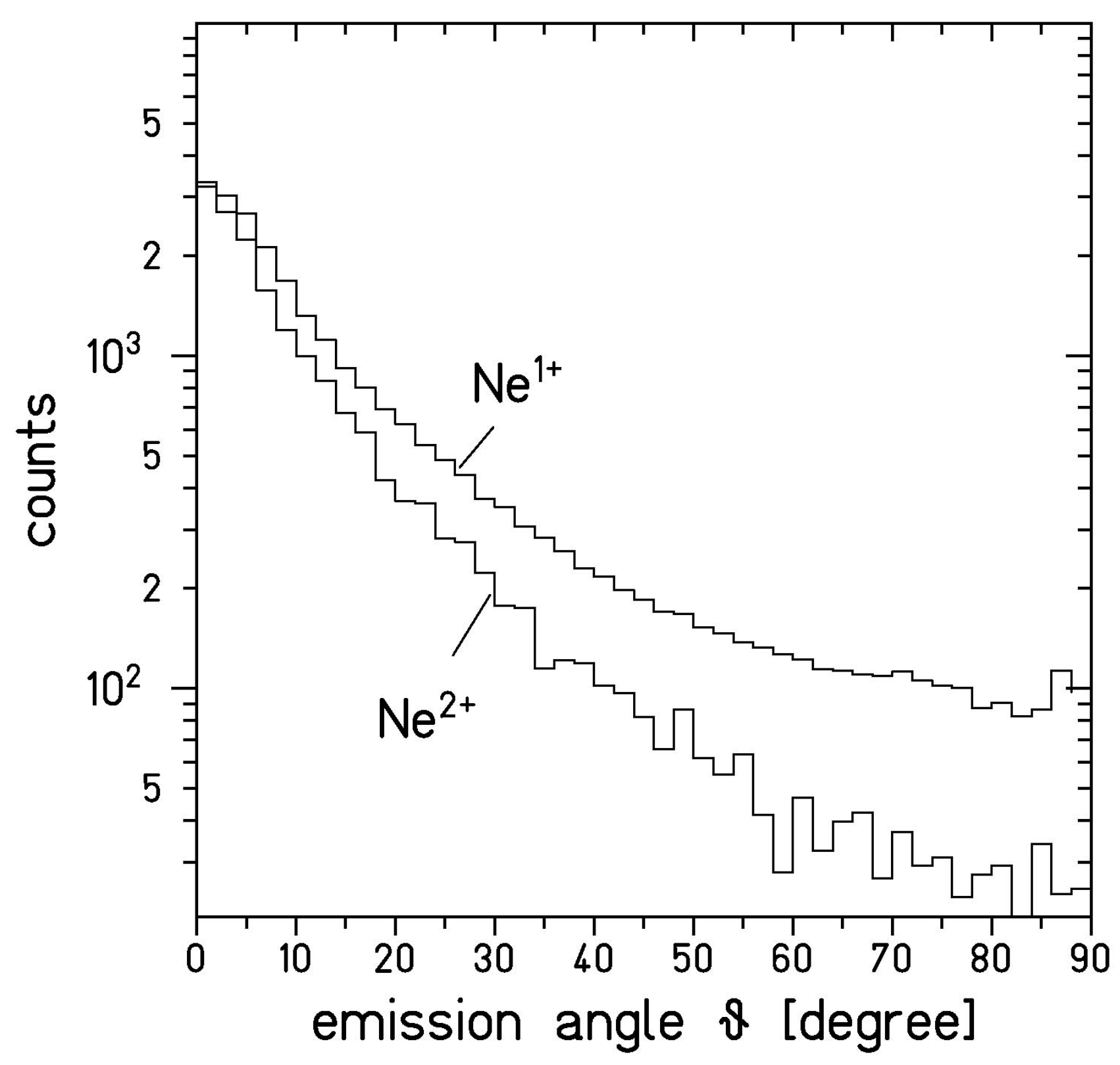

Figure 4 